\title{
Epigallocatechin gallate exerts protective effects against myocardial ischemia/reperfusion injury through the PI3K/Akt pathway-mediated inhibition of apoptosis and the restoration of the autophagic flux
}

\author{
FEIFEI XUAN ${ }^{1,2}$ and JIE JIAN ${ }^{1}$ \\ ${ }^{1}$ Department of Pharmacology, Guilin Medical University, Guilin, Guangxi 541001; \\ ${ }^{2}$ Department of Pharmacology, Guangxi Medical University, Nanning, Guangxi 530021, P.R. China
}

Received November 25, 2015; Accepted May 23, 2016

DOI: $10.3892 /$ ijmm.2016.2615

\begin{abstract}
Epigallocatechin gallate (EGCG), a polyphenol derived from green tea, exhibits a wide range of biological activities, including antioxidant, atherosclerosis and antitumor activities. In this study, the cardioprotective effects of EGCG on myocardial ischemia/reperfusion (I/R) injury in rats and the underlying mechanisms were investigated. A rat model of I/R injury was established by ligating the left anterior descending coronary artery for $30 \mathrm{~min}$, followed by reperfusion for $2 \mathrm{~h}$. The levels of I/R-induced creatine kinase-MB (CK-MB) and the release of lactate dehydrogenase (LDH), as well as the infarct size, cardiomyocyte apoptosis and cardiac functional impairment were examined and compared. Western blot analysis was carried out to elucidate the potential molecular mechanisms of action of EGCG. The results revealed that EGCG post-conditioning significantly decreased the levels of CK-MB and the release of LDH, reduced the myocardial infarct size, decreased the apoptotic rate and partially preserved heart function. Furthermore, EGCG decreased the expression of cleaved caspase- 3 concomitantly with the upregulation of PI3K, and the phosphorylation of Akt and endothelial nitric oxide synthase (eNOS). It also inhibited I/R-induced overautophagy and promoted the clearance of autophagosomes, as evidenced by a decrease in the ratio of microtubule-associated protein 1 light chain 3 (LC3)-II/LC3-I, the downregulation of Beclin1, Atg5 and p62, and the upregulation of active cathepsin D. Additionally, we observed an increase in the phosphorylation levels of the mammalian target of rapamycin (mTOR) following treatment with EGCG. Taken together, the findings of this study demonstrate that, EGCG post-conditioning alleviates
\end{abstract}

Correspondence to: Professor Jie Jian, Department of Pharmacology, Guilin Medical University, Huan Cheng North 2nd Road, Guilin, Guangxi 541004, P.R. China

E-mail: 251181281@qq.com

Key words: epigallocatechin gallate, myocardial ischemia/reperfusion, apoptosis, autophagy, PI3K/Akt myocardial I/R injury by inhibiting apoptosis and restoring the autophagic flux, which is associated with several targets of the PI3K/Akt signaling pathway.

\section{Introduction}

Myocardial infarction (MI) is a major cause of morbidity and mortality worldwide in patients with coronary heart disease (1). Early reperfusion of the ischemic region by thrombolysis or primary percutaneous coronary intervention, although effective for salvaging the damaged myocardium, can lead to further injury, such as cardiomyocyte death and cardiac dysfunction, which is known as myocardial ischemia/reperfusion (I/R) injury (2). Hence, to improve clinical outcomes in acute $\mathrm{MI}$, it is of pivotal importance to explore the safe and effective therapeutic intervention for mitigating I/R injury.

It is well established that apoptosis, a main type of programmed cell death, plays an important role in the pathogenesis of myocardial I/R injury (3). Caspases are key molecules involved in signaling cascades of programmed cell death, in which caspase- 3 has been recognized as an important initiator and promoter of apoptosis (3). Among the key survival signaling transduction pathways, the phosphoinositide 3-kinase/serine/ threonine protein kinase (PI3K/Akt) pathway plays an important role in myocardial I/R injury, and is also essential for the regulation of proliferation, differentiation and apoptosis (4). PI3K-activated Akt can regulate pro-apoptotic proteins and transcription factors, and can activate endothelial nitric oxide synthase (eNOS) to produce nitric oxide (NO), thereby promoting cell survival and protecting the heart against I/R injury (5).

Autophagy, responsible for the degradation and recycling of cellular components, is also activated in response to myocardial I/R injury. Research has shown that autophagy is a 'double-edged sword' in I/R: a slight increase in autophagy during ischemia inhibits apoptosis and promotes cell survival; however, an abnormal increase in autophagy during the reperfusion period aggravates myocardial injury (6). Autophagy is a dynamic process involving the segregation of cargo within double membrane-bound autophagosomes that fuse with and are degraded in lysosomes (7). The above process is termed 
the autophagic flux. Recent evidence has indicated that the impairment of the autophagic flux leads to the progressive consumption of cellular constituents and subsequently, to cell death in myocardial I/R injury (8). The kinase mammalian target of rapamycin (mTOR) is a key regulator of autophagy, the activity of which is enhanced by factors that activate the PI3K/Akt pathway (9).

Green tea [Camellia sinensis L. Ktze. (Theaceae)] is an extremely popular beverage that originated in China several thousands of years ago. An expanding body of evidence suggests that the consumption of green tea has several health benefits; namely, it is known to prevent or ameliorate various diseases, such as obesity, autoimmune disorders and certain types of cancer, as well as neurodegenerative and cardiovascular diseases $(10,11)$. These effects of green tea are mediated by its polyphenols known as catechins. Epigallocatechin gallate (EGCG) is the most abundant catechin found in green tea that has been shown to have antioxidant, anti-inflammatory, anti-mutagenic, anti-proliferative and anti-apoptotic effects in a variety of experimental models (12). It has been reported that EGCG has a beneficial effect on coronary artery disease (13). Previous studies have demonstrated that EGCG protects cardiomyocytes from I/R-induced apoptosis through the inhibition of signal transducer and activator of transcription (STAT)1 phosphorylation, the decrease of nuclear factor $-\kappa \mathrm{B}(\mathrm{NF}-\kappa \mathrm{B})$ and activator protein 1 expression, as well as the activation of the reperfusion injury salvage kinase (RISK) pathway (14-17). However, the mechanism responsible for the healing effects of EGCG during myocardial I/R injury have not been completely defined. In particular, the association between the autophagic flux and the therapeutic effects of EGCG in myocardial diseases have not been previously investigated, at least to the best of our knowledge.

Therefore, the aim of the present study was to investigate whether EGCG mitigates myocardial I/R injury and to further identify the potential mechanisms involved. It was hypothesized that the influence of the downstream targets of the PI3K/ Akt pathway activated by EGCG may suppress apoptosis and restore the autophagic flux, which may contribute to the cardioprotective effects of EGCG against I/R injury.

\section{Materials and methods}

Animals and experimental conditions. The experimental procedures and protocols used in this study were approved by the Ethics Committee for the Experimental Use of Animals at Guangxi Medical University (Guangxi, China) on June 18, 2015 (no. 20150618-08) and carried out in accordance with their guidelines. A total of 24 male Sprague-Dawley rats with a body weight of 250-280 g were obtained from the Guangxi Medical University Laboratory Animal Center. The rats were housed under standard conditions $\left(20-25^{\circ} \mathrm{C}, 50-60 \%\right.$ humidity, with a $12 \mathrm{~h}$ light-dark cycle) and were given standard rodent chow and free access to water.

Drugs and reagents. EGCG (Fig. 1; purity, $\geq 95 \%$ ) was obtained from Sigma-Aldrich Inc. (St. Louis, MO, USA) and diluted with saline to an appropriate concentration as needed. The creatine kinase-MB (CK-MB), lactate dehydrogenase ( $\mathrm{LDH})$ and NO detection kits were obtained from the Jiancheng Bioengineering Institute (Nanjing, China). A terminal deoxynucleotidyl transferase-mediated dUTP nick-end labeling (TUNEL) apoptosis detection kit was purchased from Roche Diagnostics (Mannheim, Germany). Antibodies recognizing PI3K (p110 $\alpha$; \#13364), Akt (\#9272), phosphorylated (p)-Akt (Ser473; \#9271), eNOS (\#9572), p-eNOS (Ser1177; \#9571), mTOR (\#2983), p-mTOR (Ser2448; \#5536), cleaved caspase-3 (\#9661), caspase-3 (\#9662), light chain 3 (LC3; \#3868), Beclin1 (\#3495), Atg5 (\#12994) and p62 (\#5114) were purchased from Cell Signaling Technology, Inc. (Danvers, MA, USA). Casthepsin D antibody (sc-10725) was purchased from Santa Cruz Biotechno logy, Inc. (Santa Cruz, CA, USA).

Experimental design. The rats were randomly assigned into 3 groups (n=8/group): i) the sham-operated group, in which the rats were subjected to surgical manipulation without ligation of the left anterior descending (LAD) coronary artery; ii) the I/R group, in which the rats were subjected to ligation of the LAD artery for $30 \mathrm{~min}$ followed by reperfusion for $2 \mathrm{~h}$ to induce I/R injury; iii) the I/R + EGCG group, in which the rats were subjected to I/R injury and administered EGCG $(10 \mathrm{mg} / \mathrm{kg}$ in saline) via a sublingual intravenous injection $10 \mathrm{~min}$ prior to the onset of reperfusion. The rats in the sham-operated and I/R model groups were administered an identical dose of normal saline to serve as a control.

General surgical procedure. The rats were anesthetized with $20 \%$ ethyl carbamate $(5 \mathrm{ml} / \mathrm{kg})$ and placed in the supine position. The chest was opened through the fourth intercostal space, and the ribs were gently retracted to expose the heart. After cutting the pericardium, the LAD coronary artery was positioned between the left atrial appendage and the pulmonary conus. A 6-0 silk suture was passed around the LAD artery, and the ends were pulled through a small vinyl tube to form a snare and then tightened. Oxygen was supplied through the trachea using an animal ventilator (respiration rate 70/ min; respiration-to-expiration ratio, 1:2; tidal volume, $50 \mathrm{ml} /$ $\mathrm{kg}$ ). Myocardial ischemia for $30 \mathrm{~min}$ was confirmed by the visual inspection of regional cyanosis of the myocardium and ST-segment elevation on an electrocardiogram. Reperfusion for $2 \mathrm{~h}$ was initiated by releasing the ligation of the LAD artery and confirmed by a color change in the ventricular surface from cyanosis to hyperemia, as previously described (18).

Assessment of cardiac function. I/R-induced cardiac dysfunction was determined by invasive hemodynamic evaluation methods. A micro-catheter connected to the MS 4000 organism signal quantitative analytical system (Longfeida Technology Co., Ltd., Shandong, China) was inserted into the left ventricle through the right common carotid artery to record left ventricular systolic pressure (LVSP), left ventricular enddiastolic pressure (LVEDP) and maximum rise/down velocity of left intraventricular pressure $\left( \pm d p / d t_{\max }\right)$ at baseline, at $30 \mathrm{~min}$ of ischemia, and after 30, 60, 90 and $120 \mathrm{~min}$ of reperfusion.

Determination of infarct size. The myocardial infarct size was measured using 2,3,5-triphenyltetrazolium chloride (TTC) staining as previously described (19). Briefly, the hearts were harvested and rinsed with normal saline. The excised left ventricle was frozen at $-80^{\circ} \mathrm{C}$ for $5 \mathrm{~min}$, and then sectioned from apex to base into approximately $2-\mathrm{mm}$-thick slices. The 
<smiles>O=C(O[C@H]1Cc2c(O)cc(O)cc2O[C@@H]1c1cc(O)c(O)c(O)c1)c1cc(O)c(O)c(O)c1</smiles>

Figure 1. Chemical structure of epigallocatechin gallate (EGCG).

slices were incubated in a solution of $1 \%$ TTC in phosphate buffered saline ( $\mathrm{pH} \mathrm{7.4)}$ at $37^{\circ} \mathrm{C}$ for $20 \mathrm{~min}$ in the dark, and then fixed in $10 \%$ formaldehyde. The slices were photographed the following day by a digital camera (Nikon, Tokyo, Japan). The infarcted (TTC non-stained) area was isolated from the rest of the cardiac tissue, which was stained red by TTC. The infarcted and normal tissues were separately weighed and the infarct size was expressed as a percentage of the mass of the left ventricle.

Measurement of cardiac enzyme levels and NO content. Blood samples were collected from the carotid artery following reperfusion, and the serum was immediately separated from the blood samples by centrifugation at 3,000 rpm for $10 \mathrm{~min}$. The levels of CK-MB and LDH content were evaluated by a colorimetric method using commercial kits (Jiancheng Bioengineering Institute) according to the manufacturer's instructions, and the absorbance was measured spectrophotometrically at a wavelength of $340 \mathrm{~nm}$ and $490 \mathrm{~nm}$, respectively. Due to its instability in physiological solutions, most of the NO is rapidly converted into nitrite $\left(\mathrm{NO}^{2-}\right)$ and further into nitrate $\left(\mathrm{NO}^{3-}\right)$. The serum levels of $\mathrm{NO}^{2-} / \mathrm{NO}^{3-}$ were measured using a $\mathrm{NO}$ detection kit according to the manufacturer's instructions. Briefly, nitrate was converted into nitrite using Aspergillus nitrite reductase, and the total nitrite level was measured using Griess reagent. The absorbance was determined at $540 \mathrm{~nm}$ using a spectrophotometer.

TUNEL assay. Cardiomyocyte apoptosis was determined using an In Situ Cell Death Detection kit, POD (Roche Diagnostics). TUNEL staining was used to quantify apoptotic cell nuclei as previously described (20). Briefly, tissue sections were washed in phosphate-buffered saline (PBS) and then fixed in a $4 \%$ paraformaldehyde solution prior to incubation in $20 \mu \mathrm{g} /$ $\mathrm{ml}$ proteinase $\mathrm{K}$ for $15 \mathrm{~min}$. After washing with PBS, the tissue sections were immersed in the TUNEL reaction mixture for $1 \mathrm{~h}$ at $37^{\circ} \mathrm{C}$ in a humid chamber. The reaction was terminated by transferring the slides to a $2 \mathrm{X}$ sodium citrate saline solution. Endogenous peroxidase activity was quenched by incubation in $0.3 \%$ hydrogen peroxide. Finally, streptavidin horseradish peroxidase was bound to the biotinylated nucleotides and the peroxidase activity was visualized in each section by the application of the stable chromogen, diaminobenzidine. In this method, the apoptotic nuclei were stained dark brown. Normal nuclei were stained blue with hematoxylin. The results were scored semi-quantitatively by averaging the number of apoptotic cells/ field at x400 magnification. Five fields were evaluated per tissue sample, and cardiomyocyte apoptosis was represented as the apoptotic index (AI) calculated as follows: $\mathrm{AI}=$ the number of TUNEL-positive cells/the total number of cells counted $x 100$.

Western blot analysis. At the end of reperfusion, a section of approximately $70 \mathrm{mg}$ of myocardial tissue was taken from the infarct area of the left ventricle. Following homogenization and protein quantification, equal amounts of protein from each sample were separated by sodium dodecyl sulfate-polyacrylamide gel electrophoresis (SDS-PAGE) and then transferred onto polyvinylidene difluoride (PVDF)-plus membranes. After blocking with 5\% bovine serum albumin (BSA), the membranes were incubated overnight at $4{ }^{\circ} \mathrm{C}$ with the following primary antibodies: PI3K (1:1,000), Akt (1:2,000), p-Akt (Ser473, 1:1,000), p-eNOS (Ser1177, 1:1,000), p-mTOR (Ser2448, 1:1,000), Atg5 (1:1,000),Beclin1 (1:1,000),LC3 (1:1,000),p62 (1:1,000) and cathepsin $\mathrm{D}(1: 500)$. The membranes were then washed 3 times in Tris-buffered saline with $0.1 \%$ Tween-20 (TBST) and incubated with the corresponding secondary antibody (anti-rabbit IgG, HRP-linked; \#7074; 1:5,000; Cell Signaling Technology, Inc.) conjugated to horseradish peroxidase at room temperature for $2 \mathrm{~h}$. Finally, the membranes were washed 3 times in TBST. Relative densitometry was performed using a computerized software package (NIH Image 1.63 software).

Statistical analysis. Each sample was assayed in triplicate. The results were averaged and expressed as the means \pm SD and data were evaluated using the Sigma Stat (version 21.0) statistical analysis program (SPSS Inc., Chicago, IL, USA). A one-way analysis of variance followed by Bonferroni's multiple comparison test was used for statistical analysis. P-values $<0.05$ were considered to indicate statistically significant differences.

\section{Results}

EGCG promotes recovery following $I / R$-induced cardiac dysfunction. No significant differences in cardiac performance were observed among the different groups prior to ischemia. During the occlusion and reperfusion process, myocardial I/R led to left ventricular dysfunction characterized by a significant increase in LVEDP, and a decrease in LVSP, $+d p /$ $d t_{\max }$ and $-d p / d t_{\max }(\mathrm{P}<0.05$ or $\mathrm{P}<0.01)$. As expected, treatment with EGCG preserved cardiac function by decreasing LVEDP, and increasing LVSP, $+d p / d t_{\max }$ and $-d p / d t_{\max }(\mathrm{P}<0.05$ or $\mathrm{P}<0.01)$ (Fig. 2 and Table I).

EGCG decreases the levels of myocardial enzyme and increases the NO content. To further evaluate and validate the protective function of EGCG during I/R injury, we measured the levels of myocardial enzymes (CK-MB and LDH) in serum (Fig. 3A and B). We found that while the I/R rats exhibited a significant increase in CK-MB and LDH levels compared with the sham-operated group $(\mathrm{P}<0.05)$, the increase in these levels was significantly abrogated in the $\mathrm{I} / \mathrm{R}+\mathrm{EGCG}$ group $(\mathrm{P}<0.05)$. In addition, compared with the I/R group, the NO content in the $\mathrm{I} / \mathrm{R}+$ EGCG group was markedly elevated $(\mathrm{P}<0.05$; Fig. $3 \mathrm{C})$. 
Table I. Effects of EGCG on hemodynamics in a rat model of I/R injury.

\begin{tabular}{|c|c|c|c|c|c|}
\hline Group & $\begin{array}{c}\text { Time } \\
(\mathrm{mmHg})\end{array}$ & LVSP & $\begin{array}{l}\text { LVEDP } \\
(\mathrm{mmHg})\end{array}$ & $\begin{array}{c}+d p / d t_{\max } \\
(\mathrm{mmHg} / \mathrm{sec})\end{array}$ & $\begin{array}{c}-d p / d t_{\max } \\
(\mathrm{mmHg} / \mathrm{sec})\end{array}$ \\
\hline \multirow[t]{6}{*}{ Sham-operated } & Baseline & $140.10 \pm 2.87$ & $1.64 \pm 0.21$ & $5529.20 \pm 320.59$ & $3731.60 \pm 283.00$ \\
\hline & I $30 \mathrm{~min}$ & $131.68 \pm 2.26$ & $1.82 \pm 0.22$ & $5395.80 \pm 309.77$ & $3667.80 \pm 333.64$ \\
\hline & $\mathrm{R} 30 \mathrm{~min}$ & $140.48 \pm 2.74$ & $1.88 \pm 0.23$ & $5514.20 \pm 211.44$ & $3557.20 \pm 340.45$ \\
\hline & $\mathrm{R} 60 \mathrm{~min}$ & $139.98 \pm 2.11$ & $1.80 \pm 0.22$ & $5470.60 \pm 282.39$ & $3586.00 \pm 252.16$ \\
\hline & $\mathrm{R} 90 \mathrm{~min}$ & $140.54 \pm 3.62$ & $1.95 \pm 0.35$ & $5469.60 \pm 329.24$ & $3840.20 \pm 320.00$ \\
\hline & $\mathrm{R} 120 \mathrm{~min}$ & $140.14 \pm 2.54$ & $2.00 \pm 0.30$ & $5512.60 \pm 217.01$ & $3543.00 \pm 363.41$ \\
\hline \multirow[t]{6}{*}{$\mathrm{I} / \mathrm{R}$} & Baseline & $139.24 \pm 1.96$ & $1.74 \pm 0.21$ & $5547.2 \pm 377.60$ & $3861.60 \pm 342.25$ \\
\hline & I $30 \mathrm{~min}$ & $134.02 \pm 2.40^{\mathrm{a}}$ & $2.72 \pm 0.36^{\mathrm{b}}$ & $4465.00 \pm 329.12^{b}$ & $3014.00 \pm 296.94^{\mathrm{a}}$ \\
\hline & $\mathrm{R} 30 \mathrm{~min}$ & $127.18 \pm 2.40^{\mathrm{b}}$ & $3.18 \pm 0.38^{b}$ & $3861.80 \pm 286.29^{b}$ & $2475.00 \pm 280.36^{\mathrm{b}}$ \\
\hline & $\mathrm{R} 60 \mathrm{~min}$ & $124.46 \pm 3.25^{\mathrm{b}}$ & $3.72 \pm 0.48^{b}$ & $3593.20 \pm 376.92^{b}$ & $2310.40 \pm 334.27^{b}$ \\
\hline & $\mathrm{R} 90 \mathrm{~min}$ & $121.58 \pm 2.67^{\mathrm{b}}$ & $3.90 \pm 0.55^{\mathrm{b}}$ & $3230.60 \pm 284.41^{\mathrm{b}}$ & $2027.80 \pm 316.04^{b}$ \\
\hline & R 120 min & $117.00 \pm 2.97^{\mathrm{b}}$ & $4.32 \pm 0.40^{\mathrm{b}}$ & $3019.60 \pm 253.87^{\mathrm{b}}$ & $1915.00 \pm 240.77^{b}$ \\
\hline \multirow[t]{6}{*}{$\mathrm{I} / \mathrm{R}+\mathrm{EGCG}$} & Baseline & $140.20 \pm 1.75$ & $1.72 \pm 0.23$ & $5511.80 \pm 381.23$ & $3814.20 \pm 263.84$ \\
\hline & I $30 \mathrm{~min}$ & $136.40 \pm 2.04$ & $2.28 \pm 0.24$ & $4921.80 \pm 263.34^{c}$ & $3333.40 \pm 365.40^{c}$ \\
\hline & $\mathrm{R} 30 \mathrm{~min}$ & $134.10 \pm 1.62^{\mathrm{d}}$ & $2.48 \pm 0.34^{\mathrm{c}}$ & $4643.20 \pm 319.91^{\mathrm{d}}$ & $3082.40 \pm 313.37^{c}$ \\
\hline & $\mathrm{R} 60 \mathrm{~min}$ & $132.68 \pm 3.23^{\mathrm{d}}$ & $2.60 \pm 0.25^{\mathrm{d}}$ & $4523.20 \pm 283.33^{\mathrm{d}}$ & $2871.80 \pm 274.12^{\mathrm{c}}$ \\
\hline & $\mathrm{R} 90 \mathrm{~min}$ & $132.00 \pm 2.45^{\mathrm{d}}$ & $2.78 \pm 0.22^{\mathrm{d}}$ & $4435.20 \pm 345.67^{\mathrm{d}}$ & $2758.00 \pm 198.41^{\mathrm{d}}$ \\
\hline & $\mathrm{R} 120 \mathrm{~min}$ & $131.02 \pm 1.83^{\mathrm{d}}$ & $2.86 \pm 0.32^{\mathrm{d}}$ & $4249.00 \pm 266.26^{\mathrm{d}}$ & $2708.00 \pm 303.62^{\mathrm{d}}$ \\
\hline
\end{tabular}

The data are presented as the means $\pm \mathrm{SD}$ ( $\mathrm{n}=8$ rats per group). ${ }^{\mathrm{P}} \mathrm{P}<0.05$ and ${ }^{\mathrm{b}} \mathrm{P}<0.01$ vs. sham-operated group; ${ }^{\mathrm{c}} \mathrm{P}<0.05$ and ${ }^{\mathrm{d}} \mathrm{P}<0.01$ vs. $\mathrm{I} / \mathrm{R}$ group. LVSP, left ventricular systolic pressure; LVEDP, left ventricular end-diastolic pressure; $\pm d p / d t_{\max }$, maximum rise/down velocity of left intraventricular pressure. I/R, ischemia/reperfusion; EGCG, epigallocatechin gallate.
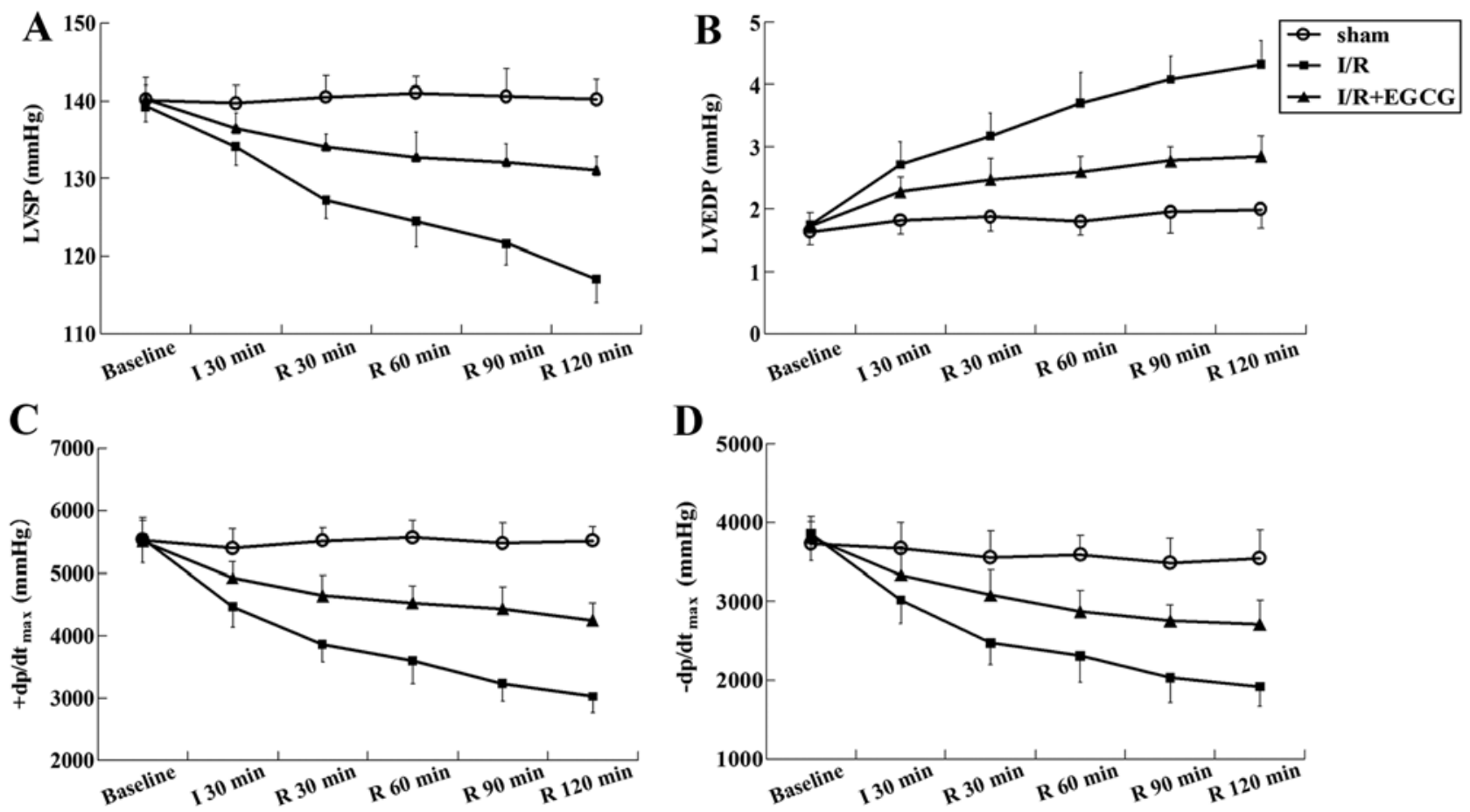

Figure 2. Effects of epigallocatechin gallate (EGCG) on left ventricular function during myocardial ischemia/reperfusion (I/R) in rats. (A) Left ventricular systolic pressure (LVSP). (B) Left ventricular end-diastolic pressure (LVEDP). (C) $+d p / d t_{\max }$. (D) $-d p / d t_{\max }$. Sham, sham-operated.

EGCG reduces the infarct size and prevents cardiomyocyte apoptosis following I/R injury. The myocardial infarct size was enlarged in the $\mathrm{I} / \mathrm{R}$ group in comparison with the sham-operated group $(\mathrm{P}<0.01$; Fig. 4). However, EGCG post-conditioning 

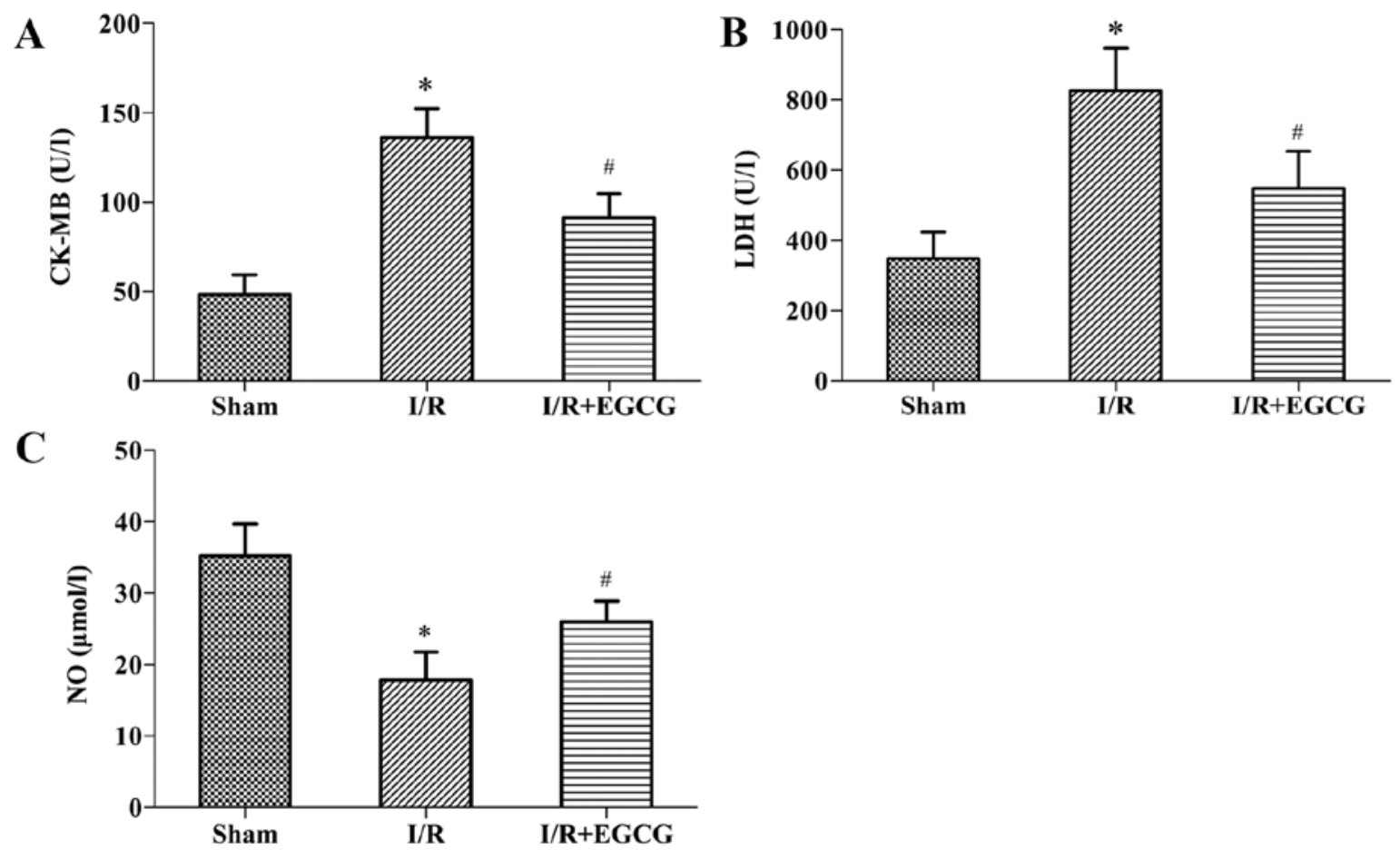

Figure 3. Effects of epigallocatechin gallate (EGCG) on the serum levels of (A) creatine kinase-MB (CK-MB), (B) lactate dehydrogenase (LDH) and (C) nitric oxide (NO). The data are presented as the means $\pm \mathrm{SD}$ ( $\mathrm{n}=8$ rats per group). ${ }^{*} \mathrm{P}<0.05$ vs. sham-operated (sham) group; ${ }^{\text {}} \mathrm{P}<0.05$ vs. I/R group.

A

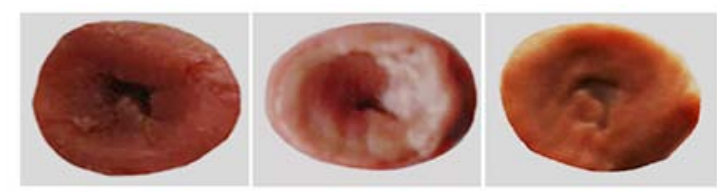

B

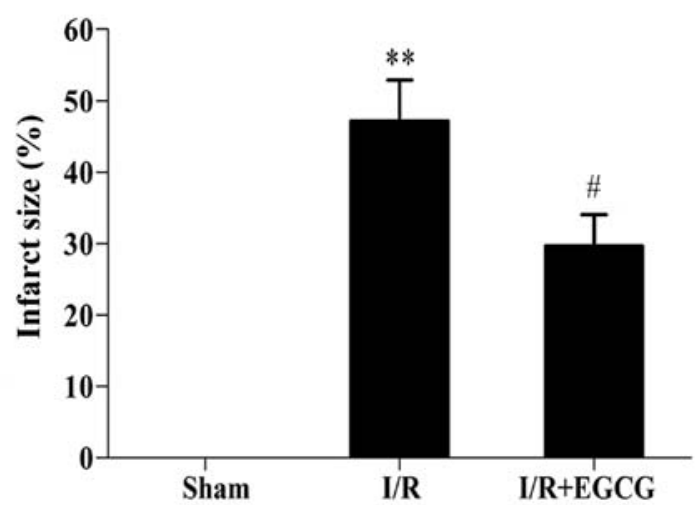

Figure 4. Effects of epigallocatechin gallate (EGCG) on the myocardial infarct size in rats subjected to ischemia/reperfusion (I/R) injury. (A) Representative images of TTC-stained sections. (B) Myocardial infarct size is expressed as the percentage of the total left ventricle volume. The data are presented as the means $\pm \mathrm{SD}$ ( $\mathrm{n}=8$ rats per group). ${ }^{* *} \mathrm{P}<0.01$ vs. sham-operated (sham) group; ${ }^{\text {}} \mathrm{P}<0.05$ vs. I/R group.

evidently decreased the infarct size when compared with the $\mathrm{I} / \mathrm{R}$ group $(\mathrm{P}<0.05)$. Furthermore, TUNEL staining revealed that apoptosis was absent in the sham-operated group. Conversely, the rats in the I/R group exhibited severe tissue damage that appeared to markedly increase the number of TUNEL-positive cells $(\mathrm{P}<0.01)$. Of note, post-conditioning with EGCG resulted in a marked reduction in the number of TUNEL-positive cells compared with the $\mathrm{I} / \mathrm{R}$ model group $(\mathrm{P}<0.05$; Fig. $5 \mathrm{~A}$ and $\mathrm{B})$. In order to explore the underlying mechanisms responsible for the anti-apoptotic effects of EGCG, the expression of caspase-3 was examined by western blot analysis. The protein expression of cleaved caspase-3 was significantly decreased following treatment with EGCG compared to the myocardial I/R model group $(\mathrm{P}<0.05$; Fig. $5 \mathrm{C}$ and $\mathrm{D})$.

EGCG restores the autophagic flux following myocardial $I / R$ injury. To investigate whether the I/R-induced myocardial injury was dependent on the impairment of the autophagic flux, the expression levels of autophagic markers, including the LC3-II/LC3-I ratio, Beclin1, Atg5 and p62, as well as the lysosomal protease, cathepsin $\mathrm{D}$, were measured by western blot analysis. Beclin1 and Atg5 are essential proteins required for the initiation of autophagosome formation (21). The conversion of LC3-I to LC3-II is closely correlated with the number of autophagosomes (33). SQSTM1/p62 is an adapter protein that links aggregated proteins in autophagosomes and promotes degradation in autolysosomes. Increased p62 levels indicate an impaired autophagic flux (22). I/R significantly increased the LC3-II/LC3-I ratio, and Beclin1, Atg5 and p62 expression compared to the sham-operated group $(\mathrm{P}<0.05$ or $\mathrm{P}<0.01)$, suggesting increased autophagic activity or an impaired autophagic flux (Fig. 6). Following treatment with EGCG, the increase in the levels of Beclin1, Atg5 and p62, and the LC3-II/LC3-I ratio were abrogated when compared with the $\mathrm{I} / \mathrm{R}$ group $(\mathrm{P}<0.05)$, indicating that EGCG prevented excessive autophagy and promoted the clearance of autophagosomes. Furthermore, an elevated level of active cathepsin $D$ was noted in the $\mathrm{I} / \mathrm{R}+\mathrm{EGCG}$ group compared with the $\mathrm{I} / \mathrm{R}$ group $(\mathrm{P}<0.05)$, indicating that EGCG restores the autophagic flux by enhancing lysosomal function. 
A

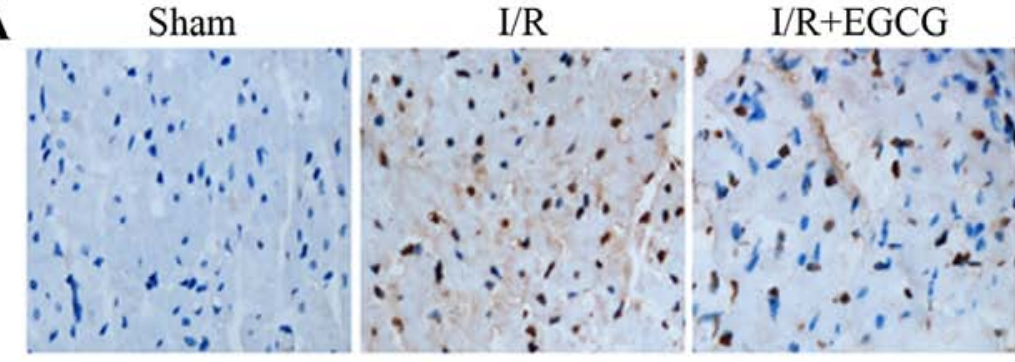

C

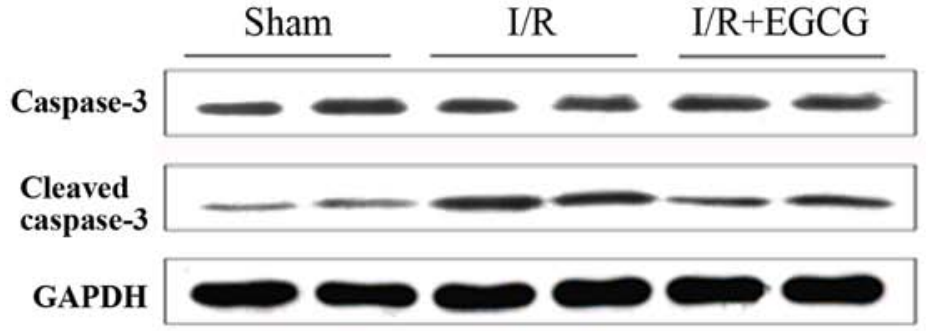

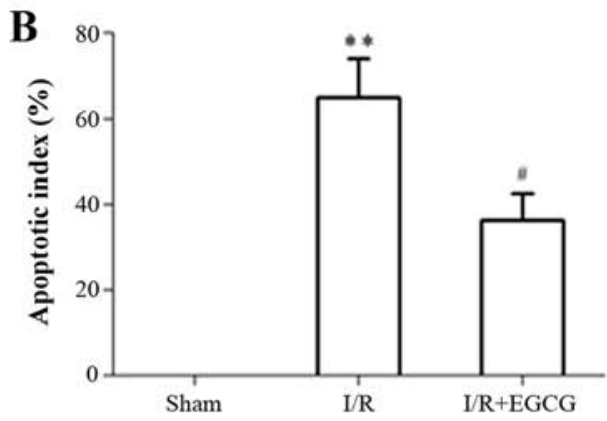

D ${ }^{2.5}$ Cleaved caspase-3

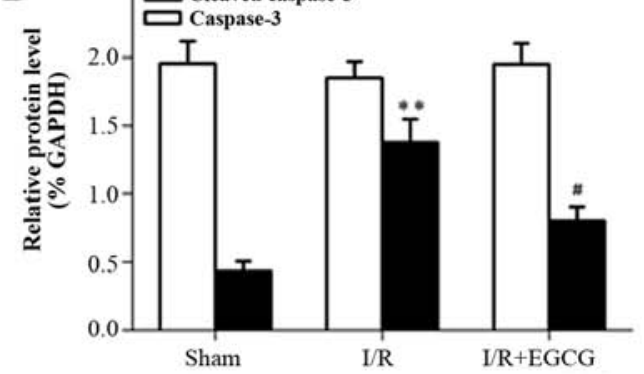

Figure 5. Effects of epigallocatechin gallate (EGCG) on myocardial apoptosis induced by ischemia/reperfusion (I/R). (A) Representative photomicrographs of TUNEL staining (x400 magnificatin). (B) Quantitative analysis of the percentage of cardiomyocyte apoptotic events. (C) Representative western blots of caspase-3 and cleaved caspase-3. (D) Quantitative analysis demonstrated the expression of caspase-3 and cleaved caspase-3. Results were normalized to glyceraldehyde 3-phosphate dehydrogenase (GAPDH). The data are presented as the means $\pm \mathrm{SD}$ ( $\mathrm{n}=8$ rats per group). ${ }^{* * *} \mathrm{P}<0.01 \mathrm{vs.} \mathrm{sham-operated} \mathrm{(sham)} \mathrm{group;}$ ${ }^{\text {"}} \mathrm{P}<0.01$ vs. I/R group.

A
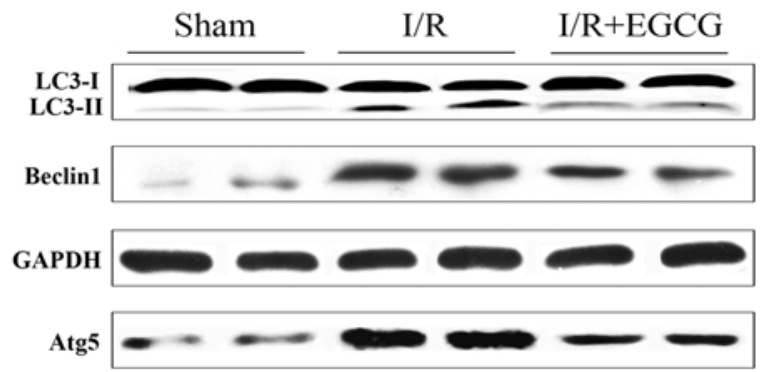

GAPDH

p62
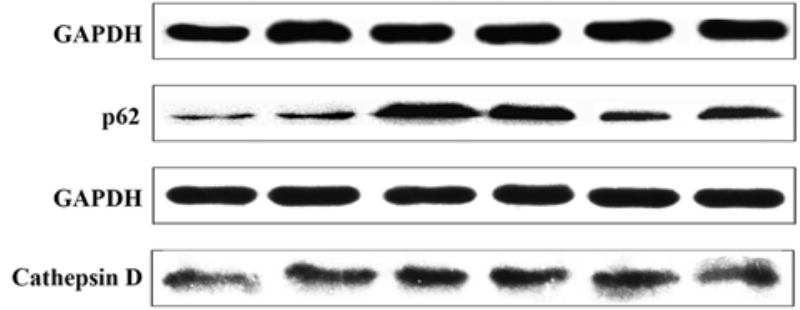

Active

$$
\begin{array}{r}
\text { Active } \\
\text { cathepsin D }
\end{array}
$$

D

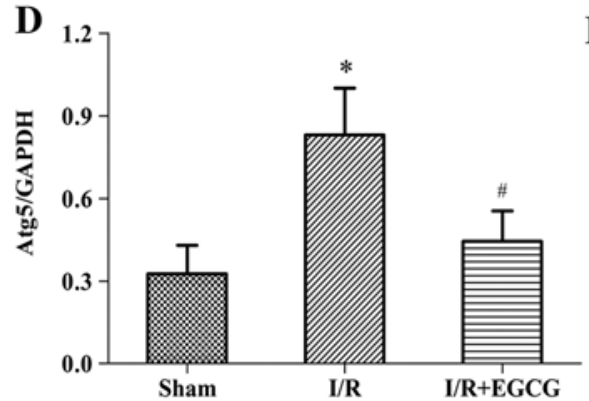

$\mathbf{E}$

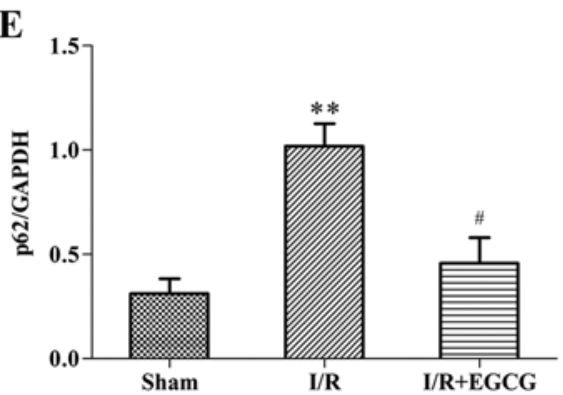

B

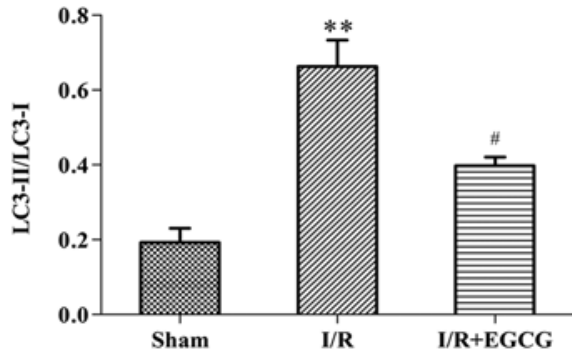

C

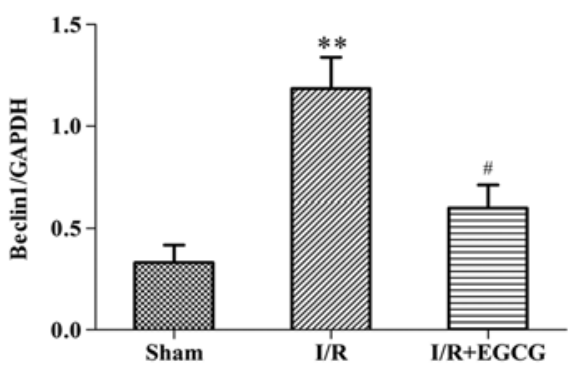

$\mathbf{F}$

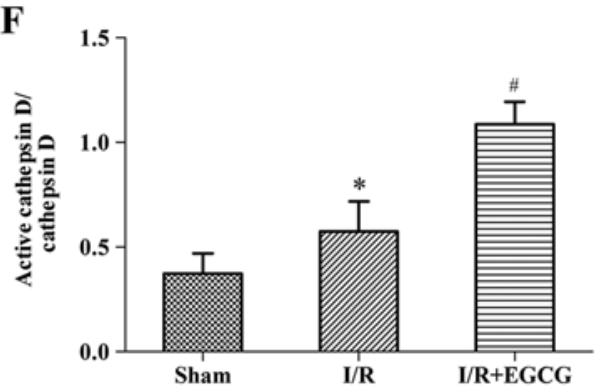

Figure 6. Epigallocatechin gallate (EGCG) restores the autophagic flux following myocardial ischemia/reperfusion (I/R) at $2 \mathrm{~h}$ of reperfusion. (A) Representative western blots of LC3-I, LC3-II, Beclin1, Atg5, p62 and cathepsin D; (B-F) Quantitative analysis demonstrated the expression of LC3-II/LC3-I ratio, Beclin1, Atg5, p62 and actived cathepsin D. The data are presented as the means $\pm \mathrm{SD}$ ( $\mathrm{n}=8$ rats per group). ${ }^{*} \mathrm{P}<0.05$ and ${ }^{* *} \mathrm{P}<0.01$ vs. sham-operated (sham) group; ${ }^{\#} \mathrm{P}<0.05$ vs. I/R group. 

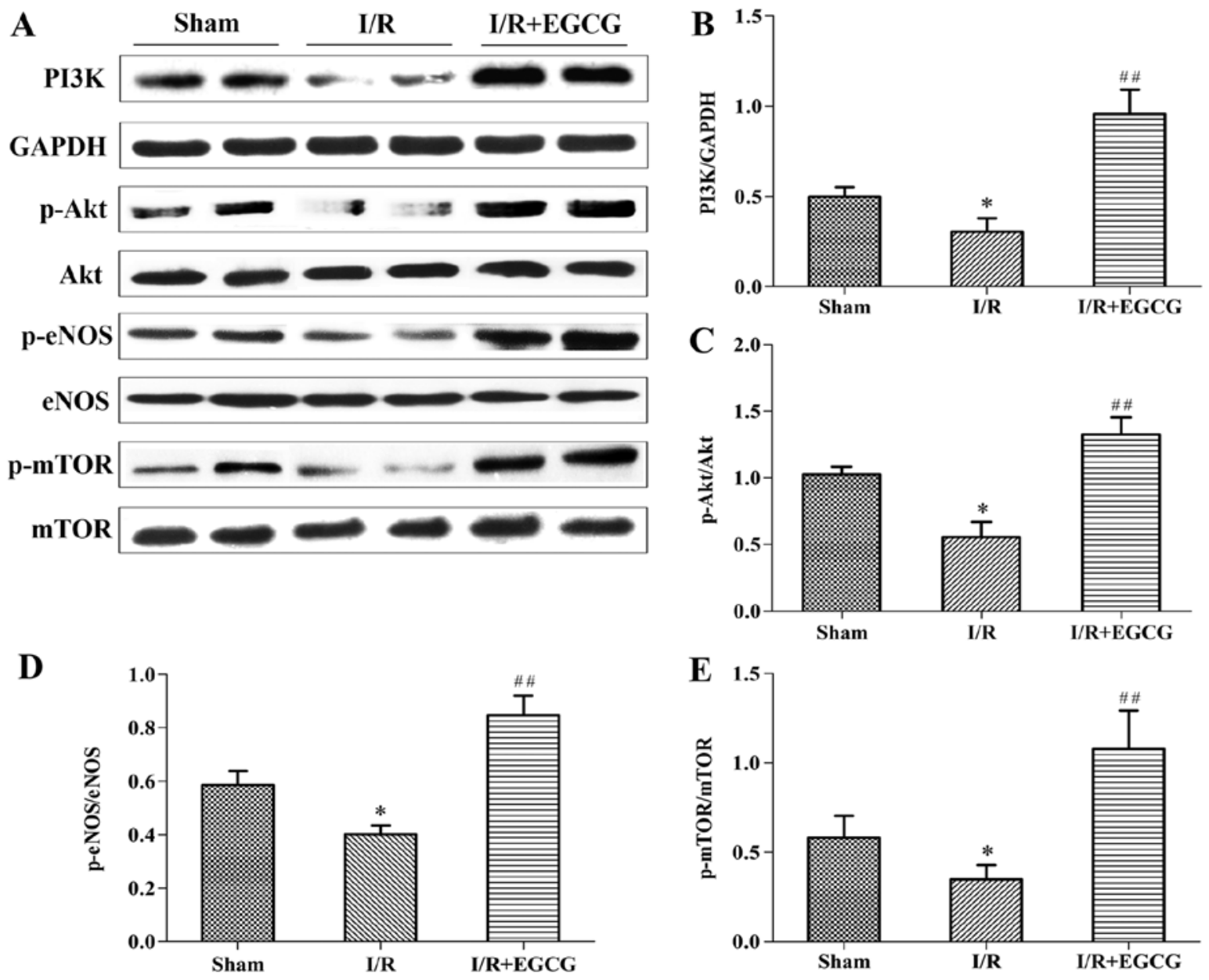

Figure 7. Epigallocatechin gallate (EGCG) enhances the expression of PI3K, p-Akt, p-endothelial nitric oxide synthase (eNOS) and p-mammalian target of rapamycin (mTOR) in the myocardium following ischemia/reperfusion (I/R). (A) Representative western blots of PI3K, p-Akt, Akt, p-eNOS, eNOS, p-mTOR and mTOR in the sham-operated (sham_, I/R and I/R + EGCG groups. (B-E) Quantitative analysis demonstrated the levels of PI3K, p-Akt, p-eNOS, and p-mTOR. Results were normalized to glyceraldehyde 3-phosphate dehydrogenase (GAPDH), total Akt, eNOS and mTOR, respectively. The data are presented as the means $\pm \mathrm{SD}$ ( $\mathrm{n}=8$ rats per group). ${ }^{*} \mathrm{P}<0.05$ vs. sham group; ${ }^{\# \#} \mathrm{P}<0.01$ vs. I/R group.

EGCG increases the expression of PI3K, $p$-Akt, p-eNOS and $p$-mTOR in the myocardium following $I / R$. In order to elucidate the role of the PI3K/Akt signaling pathway in the protective effects of EGCG against myocardial I/R injury, the expression of PI3K and p-Akt (Ser473), as well as that of the downstream targets, p-eNOS (Ser1177) and p-mTOR (Ser2448) was further investigated (Fig. 7). Compared to the sham-operated group, a significant decrease in the levels of PI3K, p-Akt, p-eNOS and p-mTOR was noted in the $\mathrm{I} / \mathrm{R}$ model group $(\mathrm{P}<0.05)$; however, a marked increase in the levels of these proteins was noted in the $\mathrm{I} / \mathrm{R}+\mathrm{EGCG}$ group $(\mathrm{P}<0.01)$. These results indicate $\mathrm{EGCG}$ may enhance the phosphorylation of eNOS and mTOR via the activation of the PI3K/Akt pathway.

\section{Discussion}

It is well known that myocardial $\mathrm{I} / \mathrm{R}$ injury results in a high proportion of cardiac dysfunction and heart failure and that no effective treatment is available to prevent this damage (2). In the present study, we confirm that EGCG post-conditioning mitigated myocardial I/R injury in rats, as manifested by a reduction of the infarct area, a decrease in the levels of cardiac enzymes and an improvement of cardiac function. These results are in accordance with those of a previous study (16). Additionally, we demonstrated that the role of EGCG in myocardial I/R recovery was related to the restoration of the autophagic flux and the inhibition of apoptosis, potentially by affecting the downstream targets of the PI3K/Akt signaling pathway.

Accumulating evidence suggests that myocardial apoptosis significantly contributes to cardiomyocyte death during $\mathrm{I} / \mathrm{R}$ injury, and blocking the apoptotic process may reduce the loss of contractile cells, minimizing myocardial I/R injury (23). It has been shown that EGCG protects cardiomyocytes against I/R-induced apoptosis in vitro and in vivo (14). Consistent with the findings of previous studies, we found that apoptosis in the group treated with EGCG, as indicated by the TUNEL-staining counting result, presented a marked reduction compared to the I/R group. Furthermore, our data lend support to the hypothesis that cleaved (activated) caspase-3 plays a pivotal role in promoting the apoptotic signaling cascade. Caspase- 3 is an established member of the caspase family that participates in the final execution phase of apoptosis (24). As shown in this study, cleaved caspase- 3 in the myocardium was widely expressed in the $\mathrm{I} / \mathrm{R}$ group, but failed to exhibit excessive activation in the rats treated with EGCG (Fig. 5). Therefore, we concluded that the inhibition of apoptosis may be one of 
the mechanisms through which EGCG exerts cardioprotective effects against I/R injury.

The PI3K/Akt pathway is known to be a target of I/R injury and plays a key role in pro-survival and anti-apoptosis (4). The activation of the PI3K/Akt signaling pathway leads to Akt phosphorylation at the Ser473 locus, producing p-Akt, and stimulates downstream targets, such as eNOS, mTOR, $\mathrm{NF}-\kappa \mathrm{B}$, the Bcl-2 family of proteins and the caspase family proteins; these then regulate apoptosis, adjust transcription factors, affect metabolism, enhance cardiomyocyte survival, and reduce the morbidity and mortality of I/R injury (25). $\mathrm{PI} 3 \mathrm{~K} / \mathrm{Akt}$ has been reported to be activated by EGCG and to mediate EGCG-induced protection against I/R injury in the heart and kidneys $(16,26)$. Among potential cytoprotective downstream targets of Akt, the phosphorylation of eNOS with the subsequent production of NO has been identified to play a critical role in PI3K/Akt-mediated protection, particularly in the anti-apoptotic effects of Akt (27). Accordingly, we further investigated the effects of EGCG post-conditioning on eNOS activity and NO production. Our data revealed that an increase in eNOS phosphorylation and NO production occurred concomitantly with the upregulation of PI3K protein and Akt phosphorylation. Thus, this suggests that EGCG protects the heart, at least in part, via the PI3K/Akt/eNOS pathway, resulting in an augmented NO content and reduced apoptosis.

In addition to apoptosis, the importance of autophagy in ischemic disease has received much attention in recent years. Autophagy is a highly regulated process in which cytosolic proteins and organelles are sequestrated by double-membrane structures of autophagosomes, transferred to lysosomes and degraded by proteases therein (28). This complete autophagic flux is essential for maintaining intracellular homeostasis and cell survival (29). Research has indicated that the myocardial I/R-induced impairment of the autophagic flux and excessive autophagy lead to cardiomyocyte death (8). Despite previous reports on how EGCG affects autophagy $(30,31)$, the mechanism through which autophagy, as a dynamic process, participate in the cardioprotective effets of EGCG remain unknown.

We hypothesized that treatment with EGCG will restore the autophagic flux during myocardial I/R, which in turn contributes to its cardioprotective effects. The process of autophagy involves nucleation, autophagosome formation, the fusion of autophagosomes to lysosomes, and degradation in lysosomes (28). Beclin1 forms a complex with class III PI3K and is localized to the pre-autophagosomal structure mediating vesicle nucleation (32). The formation of the autophagosome is initiated by the Atg12-Atg5-Atg16 complex and LC3-Iphospholipid conjugates (LC3-II) (33). Thus, the increased ratio of LC3-II/LC3-I, Beclin1 and Atg5 are markers for autophagosome formation (34). Cathepsin D is a major lysosomal protease that degrades materials delivered to lysosomes. The inhibition of cathepsin D activity may compromise lysosomal function, thus blocking the fusion of autophagosomes with lysosomes, increasing the accumulation of autophagosomes (35). Moreover, the p62 protein is a well-known autophagy substrate, which upon direct binding to LC3-II incorporates into autophagosomes and is efficiently degraded by autophagy (36). Accordingly, when the autophagic degradation pathway is blocked, p62 accumulation occurs. In this study, myocardial I/R increased the LC3-II/ LC3-I ratio, and upregulated the protein expression of Beclin1,
Atg5 and p62 compared to the sham-operated rats. These results indicated that myocardial I/R led to the impairment of the autophagic flux by inducing autophagosome formation, but impairing autophagosome clearance. In support of our hypothesis, EGCG restored the I/R-impaired autophagy flux, which was characterized by a decrease in the protein expression levels of the autophagy proteins, Beclin1, Atg5 and p62, and the LC3-II/LC3-I ratio, as well as an increase in the level of active cathepsin D.

It has been shown that the PI3K/Akt signaling pathway is involved in the cardioprotection of EGCG (16). The kinase mammalian target of rapamycin, mTOR, is an important downstream target of the PI3K/Akt pathway, which is positively regulated by $\mathrm{PI} 3 \mathrm{~K} / \mathrm{Akt}$ and results in the inhibition of autophagy (37). Previous studies have demonstrated that phosphorylated mTOR provides cardioprotection by restoring the impaired autophagic flux and enhancing recovery in myocardial I/R injury (38). Coincidently, the present study demonstrated that EGCG inhibited the over-activation of autophagy and promoted autophagosome clearance, accompanied by the upregulation of both p-Akt and p-mTOR. Since the activation of mTOR signaling is known to inhibit autophagic activity and improve autophagic flux $(39,40)$, our data suggest that EGCG attenuates I/R-induced excessive autophagy and restores the autophagic flux through the PI3K/Akt/mTOR pathway.

Autophagy has been demonstrated to engage in a complex interplay with apoptosis. Recently, interconnections between the autophagic and the apoptotic pathways, as well as their alternative functions in cell survival or death have been demonstrated (7). For instance, an autophagic flux inhibitor induces the activation of caspases and the apoptosis of cardiomyocytes (8). Atg5 depletion promotes apoptosis (41). In the present study, EGCG restored the autophagic flux and simultaneously inhibited cardiomyocyte apoptosis. Nevertheless, further investigations are warranted in order to elucidate the interactions between the underlying mechanisms responsible for the autophagic flux and apoptosis.

In conclusion, treatment with EGCG effectively protected the rat hearts from I/R injury in vivo. This effect of EGCG may be related to the inhibition of apoptosis and the restoration of the autophagic flux via the regulation of several downstream molecules of the PI3K/Akt signaling pathway.

However, there are some limitations to the present study. Firstly, the autophagic flux needs to be further evaluated by comparing the extent of autophagosome accumulation in the presence of inhibitors of lysosomal fusion or degradation or both. Secondly, our data manifested that the inhibition of apoptosis and the restoration of the autophagic flux were involved in the cardioprotective effects of EGCG; however, further studies are required in order to clarify the causal link between these mechanisms.

\section{Acknowledgements}

This study was supported by the National Natural Science Foundation of China (no. 81360041), the Project of Natural ScienceFoundation of Guangxi,China(2012GXNSFAA053148) and by the Guangxi College of Higher Education Outstanding Talent project. 


\section{References}

1. Finegold JA, Asaria P and Francis DP: Mortality from ischaemic heart disease by country, region, and age: Statistics from World Health Organisation and United Nations. Int J Cardiol 168: 934-945, 2013

2. Hausenloy DJ and Yellon DM: Myocardial ischemia-reperfusion injury: A neglected therapeutic target. J Clin Invest 123: 92-100, 2013.

3. Ganesan R, Mittl PR, Jelakovic S and Grütter MG: Extended substrate recognition in caspase-3 revealed by high resolution X-ray structure analysis. J Mol Biol 359: 1378-1388, 2006.

4. Ha T, Hu Y, Liu L, Lu C, McMullen JR, Kelley J, Kao RL, Williams DL, Gao X and Li C: TLR2 ligands induce cardioprotection against ischaemia/reperfusion injury through a PI3K/Akt-dependent mechanism. Cardiovasc Res 87: 694-703, 2010.

5. Ou HC, Lee WJ, Lee SD, Huang CY, Chiu TH, Tsai KL, Hsu WC and Sheu WH: Ellagic acid protects endothelial cells from oxidized low-density lipoprotein-induced apoptosis by modulating the PI3K/Akt/eNOS pathway. Toxicol App Pharmacol 248: 134-143, 2010.

6. Wei K, Wang P and Miao CY: A double-edged sword with therapeutic potential: An updated role of autophagy in ischemic cerebral injury. CNS Neurosci Ther 18: 879-886, 2012.

7. Zhang YL, Yao YT, Fang NX, Zhou CH, Gong JS and Li LH Restoration of autophagic flux in myocardial tissues is required for cardioprotection of sevoflurane postconditioning in rats. Acta Pharmacol Sin 35: 758-769, 2014.

8. Ma X, Liu H, Foyil SR, Godar RJ, Weinheimer CJ, Hill JA and Diwan A: Impaired autophagosome clearance contributes to cardiomyocyte death in ischemia/reperfusion injury. Circulation 125 : 3170-3181, 2012

9. Xiao J, Zhu X, Ji G, Yang Q, Kang B, Zhao J, Yao F, Wu L, $\mathrm{Ni} X$ and Wang Z: Ulinastatin protects cardiomyocytes against ischemia reperfusion injury by regulating autophagy through mTOR activation. Mol Med Rep 10: 1949-1953, 2014.

10. Pae $\mathrm{M}$ and $\mathrm{Wu} \mathrm{D}$ : Immunomodulating effects of epigallocatechin-3-gallate from green tea: Mechanisms and applications. Food Funct 4: 1287-1303, 2013.

11. Zeng X, Li Q, Zhang M, Wang W and Tan X: Green tea may be benefit to the therapy of atrial fibrillation. J Cell Biochem 112: $1709-1712,2011$

12. Chen L and Zhang HY: Cancer preventive mechanisms of the green tea polyphenol (-)-epigallocatechin-3-gallate. Molecules 12: 946-957, 2007.

13. Widlansky ME, Hamburg NM, Anter E, Holbrook M, Kahn DF Elliott JG, Keaney JF Jr and Vita JA: Acute EGCG supplementation reverses endothelial dysfunction in patients with coronary artery disease. J Am Coll Nutr 26: 95-102, 2007.

14. Townsend PA, Scarabelli TM, Pasini E, Gitti G, Menegazzi M, Suzuki H, Knight RA, Latchman DS and Stephanou A: Epigallocatechin-3-gallate inhibits STAT-1 activation and protects cardiac myocytes from ischemia/reperfusion-induced apoptosis. FASEB J 18: 1621-1623, 2004.

15. Aneja R, Hake PW, Burroughs TJ, Denenberg AG, Wong HR and Zingarelli B: Epigallocatechin, a green tea polyphenol, attenuates myocardial ischemia reperfusion injury in rats. Mol Med 10: 55-62, 2004.

16. Kim SJ, Li M, Jeong CW, Bae HB, Kwak SH, Lee SH, Lee HJ, Heo BH, Yook KB and Yoo KY: Epigallocatechin-3-gallate, a green tea catechin, protects the heart against regional ischemiareperfusion injuries through activation of RISK survival pathways in rats. Arch Pharm Res 37: 1079-1085, 2014.

17. Piao CS, Kim DS, Ha KC, Kim HR, Chae HJ and Chae SW: The protective effect of epigallocatechin-3 gallate on ischemia/reperfusion injury in isolated rat hearts: An ex vivo Approach. Korean J Physiol Pharmacol 15: 259-266, 2011.

18. Ahmed LA, Salem HA, Attia AS and Agha AM: Pharmacological preconditioning with nicorandil and pioglitazone attenuates myocardial ischemia/reperfusion injury in rats. Eur J Pharmacol 663: 51-58, 2011.

19. Yao C, Shi X, Lin X, Shen L, Xu D and Feng Y: Increased cardiac distribution of mono-PEGylated Radix Ophiopogonis polysaccharide in both myocardial infarction and ischemia/reperfusion rats. Int J Nanomedicine 10: 409-418, 2015.

20. Wu N, Li W, Shu W and Jia D: Protective effect of picroside II on myocardial ischemia reperfusion injury in rats. Drug Des Devel Ther 8: 545-554, 2014.
21. Ohsumi Y: Molecular dissection of autophagy: two ubiquitin-like systems. Nat Rev Mol Cell Biol 2: 211-216, 2001.

22. Bjørkøy G, Lamark T, Pankiv S, Øvervatn A, Brech A and Johansen T: Monitoring autophagic degradation of p62/SQSTM1. Methods Enzymol 452: 181-197, 2009.

23. Liu H, Guo X, Chu Y and Lu S: Heart protective effects and mechanism of quercetin preconditioning on anti-myocardial ischemia reperfusion (IR) injuries in rats. Gene 545: 149-155, 2014.

24. Giakoustidis DE, Giakoustidis AE, Iliadis S, Koliakou K, Antoniadis N, Kontos N, Papanikolaou V, Papageorgiou G, Kaldrimidou E and Takoudas D: Attenuation of liver ischemia/ reperfusion induced apoptosis by epigallocatechin-3-gallate via down-regulation of NF-kappaB and c-Jun expression. J Surg Res 159: 720-728, 2010

25. Yao H, Han X and Han X: The cardioprotection of the insulinmediated PI3K/Akt/mTOR signaling pathway. Am J Cardiovasc Drugs 14: 433-442, 2014.

26. Lv J, Feng M, Zhang L, Wan X, Zeng YC, Liang PF and Xu AP: Protective effect of epigallocatechin gallate, a major constituent of green tea, against renal ischemia-reperfusion injury in rats. Int Urol Nephrol 47: 1429-1435, 2015.

27. Deng C, Sun Z, Tong G, Yi W, Ma L, Zhao B, Cheng L, Zhang J, Cao F and Yi D: $\alpha$-Lipoic acid reduces infarct size and preserves cardiac function in rat myocardial ischemia/reperfusion injury through activation of PI3K/Akt/Nrf2 pathway. PLoS One 8: e58371, 2013

28. Hariharan N, Zhai P and Sadoshima J: Oxidative stress stimulates autophagic flux during ischemia/reperfusion. Antioxid Redox Signal 14: 2179-2190, 2011

29. Xie H, Xu Q, Jia J, Ao G, Sun Y, Hu L, Alkayed NJ, Wang C and Cheng J: Hydrogen sulfide protects against myocardial ischemia and reperfusion injury by activating AMP-activated protein kinase to restore autophagic flux. Biochem Biophys Res Commun 458: 632-638, 2015

30. Li W, Zhu S, Li J, Assa A, Jundoria A, Xu J, Fan S, Eissa NT, Tracey KJ, Sama AE and Wang H: EGCG stimulates autophagy and reduces cytoplasmic HMGB1 levels in endotoxin-stimulated macrophages. Biochem Pharmacol 81: 1152-1163, 2011.

31. Kim HS, Montana V, Jang HJ, Parpura V and Kim JA: Epigallocatechin gallate (EGCG) stimulates autophagy in vascular endothelial cells: A potential role for reducing lipid accumulation. J Biol Chem 288: 22693-22705, 2013.

32. Kang R, Zeh HJ, Lotze MT and Tang D: The Beclin 1 network regulates autophagy and apoptosis. Cell Death Differ 18: 571-580, 2011.

33. Herzog C, Yang C, Holmes A and Kaushal GP: zVAD-fmk prevents cisplatin-induced cleavage of autophagy proteins but impairs autophagic flux and worsens renal function. Am J Physiol Renal Physiol 303: F1239-F1250, 2012.

34. Weidberg H, Shvets E and Elazar Z: Biogenesis and cargo selectivity of autophagosomes. Annu Rev Biochem 80: 125-156, 2011.

35. Tatti M, Motta M, Di Bartolomeo S, Scarpa S, Cianfanelli V, Cecconi F and Salvioli R: Reduced cathepsins B and D cause impaired autophagic degradation that can be almost completely restored by overexpression of these two proteases in Sap C-deficient fibroblasts. Hum Mol Genet 21: 5159-5173, 2012.

36. Mizushima N, Yoshimori T and Levine B: Methods in mammalian autophagy research. Cell 140: 313-326, 2010.

37. Wang ZG, Wang Y, Huang Y, Lu Q, Zheng L, Hu D, Feng WK, Liu YL, Ji KT, Zhang HY, et al: bFGF regulates autophagy and ubiquitinated protein accumulation induced by myocardial ischemia/reperfusion via the activation of the PI3K/Akt/mTOR pathway. Sci Rep 5: 9287, 2015.

38. Sciarretta S, Volpe M and Sadoshima J: Mammalian target of rapamycin signaling in cardiac physiology and disease. Circ Res 114: 549-564, 2014

39. Hu YY, Zhou CH, Dou WH, Tang W, Hu CY, Hu DM, Feng H, Wang JZ, Qian MJ, Cheng GL and Wang SF: Improved autophagic flux is correlated with mTOR activation in the later recovery stage of experimental acute pancreatitis. Pancreatology 15: 470-477, 2015.

40. Jung CH, Ro SH, Cao J, Otto NM and Kim DH: mTOR regulation of autophagy. FEBS Lett 584: 1287-1295, 2010.

41. Yue W, Hamaï A, Tonelli G, Bauvy C, Nicolas V, Tharinger H, Codogno P and Mehrpour M: Inhibition of the autophagic flux by salinomycin in breast cancer stem-like/progenitor cells interferes with their maintenance. Autophagy 9: 714-729, 2013. 